\title{
Geometric phase gate based on the ac Stark shift
}

\author{
Mahmood Irtiza Hussain, ${ }^{1}$ Ebubechukwu O. Ilo-Okeke, ${ }^{2,3}$ and Tim Byrnes ${ }^{3}$ \\ ${ }^{1}$ Centre for Quantum Dynamics, Griffith University, Nathan, Queensland 4111, Australia \\ ${ }^{2}$ Department of Physics, Federal University of Technology, P. M. B. 1526, Owerri, Imo State, Nigeria \\ ${ }^{3}$ National Institute of Informatics, 2-1-2 Hitotsubashi, Chiyoda-Ku, Tokyo 101-8430, Japan
}

(Dated: October 2, 2015)

\begin{abstract}
We present an alternate derivation of the geometric phase gate based on the ac Stark shift for two qubits coupled to a shared cavity mode. Our derivation differs from the standard one in that the qubit-light coupling occurs purely as an ac Stark shift, rather than a state-dependent force. We derive expressions for the geometric phases induced on the qubit states arising from the displacement of coherent light. Simple analytic expressions are obtained for sinusodial driving of the cavity field.
\end{abstract}

PACS numbers:

\section{INTRODUCTION}

Quantum correlations are of utmost importance and one of the indispensable ingredients of quantum information processing [1-5]. Production of fast and robust two or multi qubit gates are ultimate goals for storing and executing quantum information [6]. The inevitable interaction between system and its environment (reservoir) makes the quantum states fragile. This feature of quantum states (systems) impairs their performance when used as quantum logic gates, and hence may limit their application for quantum information. Several schemes have been suggested to mitigate this problem. Geometric quantum computation is one of the proposal to achieve fault tolerant quantum computation [7]. In geometric quantum computation information is encoded on the energy levels of the atoms and a quantum gate is used to drive the atom along a cyclic path or in a closed loop. In doing so, the atomic states accumulate geometric phases [8] in addition to a dynamical phase. Because geometric phases are robust against environmental fluctuations, they have found applications in the geometric phase gate for fault tolerant computation. These have been realised and studied in systems such as ions under state dependent forces [9-14], quantum dots [15-17], semiconductors materials [18], microwave potentials [19], nuclear magnetic resonance [20-22], and superconducting materials [23-25].

In this paper we consider an alternate derivation of the geometric phase gate for two qubits interacting with a common bosonic mode. Specifically, we consider the so-called "unconventional" geometric phase gate where the bosonic mode is manipulated in order to produce entanglement at the end of the operation. In the standard derivation $[10,12]$, the form of the interaction between the optical mode and the qubits is a state-dependent force, of the form

$$
H_{\text {int }} \propto\left(a+a^{\dagger}\right)\left(\sigma_{1}^{z}+\sigma_{2}^{z}\right)
$$

where $a$ is the annihilation operator for the bosonic mode and $\sigma_{i}^{z}$ are the Pauli operators for each of the qubits labeled by $i=1,2$. Instead of the state-dependent force,
FIG. 1: Schematic experimental configuration for the geometric phase gate. Two qubits are placed in a cavity such that an ac Stark shift occurs on the energy levels. The geometric phase gate then performed by the following procedure. A laser is applied to the cavity such that both qubits are illuminated, and controlled such as to follow the evolution $|\alpha(t)\rangle$ for a time $t=[0, T]$. The phase (10) is induced at the end of the evolution.

in this paper we consider a coupling of the form of an ac Stark shift

$$
H_{\text {int }} \propto a^{\dagger} a\left(\sigma_{1}^{z}+\sigma_{2}^{z}\right) .
$$

We show that this type of interaction between the qubits and the bosonic field can be equally used to implement the geometric phase gate. Our derivation follows the methods presented in Ref. [12], based on an exact solution to the dynamics of the coherent bosonic field under a time-dependent displacement. Exact expressions for the phase picked up by the qubit states are derived, and an example solution is given for the necessary conditions of the evolution.

This paper is organized as follows. In Sec. II we present a modified derivation of the geometric phase gate using the ac Stark shift rather than state-dependent forces. In Sec. III we describe a concrete implementation of the optical pulses that satisfy the necessary conditions described in Sec. II. In Sec. IV we show an experimental implementation for our proposed scheme, discussing which energy levels are used. We finally conclude the results of the paper in Sec. V.

\section{GEOMETRIC PHASE GATE WITH AC STARK SHIFTS}

Let us consider that we have the following Hamiltonian available between two qubits

$$
\begin{aligned}
H= & \hbar \omega_{0} a^{\dagger} a+G\left(\sigma_{1}^{z}+\sigma_{2}^{z}\right) a^{\dagger} a \\
& -\frac{F(t)}{\sqrt{2}}\left(a e^{i\left(\omega_{0}-\Delta\right) t}+a^{\dagger} e^{-i\left(\omega_{0}-\Delta\right) t}\right) .
\end{aligned}
$$


Here $a$ is an annihilation operator for a bosonic mode, $\sigma_{1,2}^{z}$ are Pauli $z$-operators for qubits 1 and 2 respectively. The first term in (3) gives the energy of the bosonic mode $\hbar \omega_{0}$. The second is a diagonal coupling of magnitude $G$ between the bosonic and qubit degrees of freedom. For an implementation with cold atoms, this may come from an ac Stark shift of the atomic levels due to the bosonic field. The last term is the displacement operator for the bosonic mode that is controllable with a time-dependent coefficient $F(t)$. In the rotating frame $a_{r}=a e^{i \omega_{0} t}$ the Hamiltonian is

$$
H=G\left(\sigma_{1}^{z}+\sigma_{2}^{z}\right) a_{r}^{\dagger} a_{r}-\frac{F(t)}{\sqrt{2}}\left(a_{r} e^{-i \Delta t}+a_{r}^{\dagger} e^{i \Delta t}\right)
$$

such that relative to the phase of the optical field the force term $F(t)$ is rotating with a detuned frequency $\Delta$.

The precise physical realization of (3) will be described in section IV. The basic idea of the scheme may be understood as given in Figure 1. Two qubits are placed in a cavity and illuminated with the same laser field, in a time varying coherent state $|\alpha(t)\rangle$. The laser is far detuned from the transition to an excited state of the qubit, such that there is an ac Stark shift to the energy level, giving rise to the second term in (3). The scheme then illuminates the two qubits with an initial coherent state $|\alpha(0)\rangle=\left|\alpha_{0}\right\rangle$, and is moved through phase space of $(\operatorname{Re}(\alpha), \operatorname{Im}(\alpha))$ such that the state at the end of the evolution is the same as the initial state $|\alpha(T)\rangle=e^{i \Phi(T)}\left|\alpha_{0}\right\rangle$, where $\Phi(T)$ is a phase that depends upon the state of the qubits (see Figure 2). Depending on the state of the qubits, different phases are picked up, thus creating entanglement between the qubits. A similar configuration of qubits in cavities was proposed in Ref. [27] although our derivation of the geometric phases does not follow the same approach.

Following Ref. [12], we may derive the effective phase generated by the above procedure. The Heisenberg equations of motion for the bosonic mode is

$$
\frac{d a}{d t}=-\frac{i}{\hbar}\left[\left(\hbar \omega_{0}+G \sigma_{1}^{z}+G \sigma_{2}^{z}\right) a-\frac{F(t)}{\sqrt{2}} e^{-i\left(\omega_{0}-\Delta\right) t}\right] .
$$

This may be explicitly solved to give

$$
a(t)=e^{-i \Omega t}\left[a(0)+\frac{i}{\sqrt{2} \hbar} \int_{0}^{t} e^{i \Omega \tau} F(\tau) d \tau\right]
$$

where

$$
\Omega=\Delta+G\left(\sigma_{1}^{z}+\sigma_{2}^{z}\right) / \hbar .
$$

Starting from an initial coherent state $\left|\alpha_{0}\right\rangle$, the above creates a time dependent dispacement $|\alpha(t)\rangle$ according to

$$
\alpha(t)=e^{-i \Omega t}\left[\alpha_{0}+\frac{i}{\sqrt{2} \hbar} \int_{0}^{t} e^{i \Omega \tau} F(\tau) d \tau\right] .
$$

We consider the force $F(t)$ to be applied for a time $T$, at the end of which we demand that the coherent state be
FIG. 2: Displacement of coherent field $|\alpha(t)\rangle$ under the force $F(t)$.

returned to its initial state $|\alpha(T)\rangle=\left|\alpha_{0}\right\rangle$. Thus we have the condition

$$
\int_{0}^{T} e^{i \Omega \tau} F(\tau) d \tau=0
$$

Following the same derivation as in Ref. [10, 12], the phase picked up by the coherent state is

$$
\Phi(T)=\frac{1}{2 \hbar^{2}} \operatorname{Im} \int_{0}^{T} d \tau_{1} \int_{0}^{\tau_{1}} d \tau_{2} e^{i \Omega \tau_{r}} F\left(\tau_{1}\right) F\left(\tau_{2}\right) .
$$

where $\tau_{r}=\tau_{1}-\tau_{2}$. The exponential term in the above equation may be expanded and we obtain the following expression,

$$
\Phi_{\text {qubit }}(T)=\phi_{0}+\phi_{1}\left(\sigma_{1}^{z}+\sigma_{2}^{z}\right)+\phi_{2} \sigma_{1}^{z} \sigma_{2}^{z}
$$

where

$$
\begin{aligned}
& \phi_{0}=\frac{1}{2 \hbar^{2}} \int_{0}^{T} d \tau_{1} \int_{0}^{\tau_{1}} d \tau_{2} \sin \left(\Delta \tau_{r}\right) \cos ^{2}\left(\frac{G \tau_{r}}{\hbar}\right) F\left(\tau_{1}\right) F\left(\tau_{2}\right), \\
& \phi_{1}=\frac{1}{4 \hbar^{2}} \int_{0}^{T} d \tau_{1} \int_{0}^{\tau_{1}} d \tau_{2} \sin \left(\frac{2 G \tau_{r}}{\hbar}\right) \cos \left(\Delta \tau_{r}\right) F\left(\tau_{1}\right) F\left(\tau_{2}\right) \\
& \phi_{2}=-\frac{1}{2 \hbar^{2}} \int_{0}^{T} d \tau_{1} \int_{0}^{\tau_{1}} d \tau_{2} \sin \left(\Delta \tau_{r}\right) \sin ^{2}\left(\frac{G \tau_{r}}{\hbar}\right) F\left(\tau_{1}\right) F\left(\tau_{2}\right) .
\end{aligned}
$$

In our unconventional geometric phase gates the geometric and dynamical phases are proportional. We are not going into details of this proportionality, since it has been explicitly explained in [12]. We see that the phase includes a term proportional to $\sigma_{1}^{z} \sigma_{2}^{z}$, which produces an entangling gate as desired. In addition we have constant and terms proportional to $\sigma_{i}^{z}$, which give an global phase and single qubit rotations respectively. Assuming single qubit control of the qubits are possible, the $\phi_{1}$ terms may be cancelled off as desired by application of the reverse rotations which cancel these factors.

\section{EXAMPLE SOLUTION}

We now discuss a concrete example for implementing the two qubit entanglement. Unlike the formulation of Ref. $[10,12], \Omega$ is state dependent so we now give an example of how (8) can be satisfied. Assuming that the detuning $\Delta$ can be freely chosen, let us select the condition

$$
\hbar \Delta=2 G n
$$

where $n$ is an integer. The frequencies that (6) may take are then

$$
\hbar \Omega=2 G(n-1), 2 G n, 2 G(n+1)
$$


Noting that all the frequencies are even multiples of $G / \hbar$, (8) may then be satisfied by choosing

$$
\begin{aligned}
F(\tau) & =F_{0} \sin (G m \tau / \hbar) \\
T & =\frac{2 \pi \hbar}{G}
\end{aligned}
$$

where $m$ is an odd integer and $F_{0}$ is an energy amplitude that may be freely chosen.

The phases induced by geometric phase gates may then be evaluated exactly to give

$$
\begin{aligned}
& \phi_{0}=-\frac{F_{0}^{2} n \pi}{G^{2}}\left(\frac{8-2 m^{2}-24 n^{2}+\left(m^{2}-4 n^{2}\right)^{2}}{\left(m^{2}-4 n^{2}\right) M}\right) \\
& \phi_{1}=-\frac{F_{0}^{2} \pi}{G^{2} 2}\left(\frac{\sqrt{M+(4 m n)^{2}}}{M}\right) \\
& \phi_{2}=-\frac{F_{0}^{2} 2 n \pi}{G^{2}}\left(\frac{4 n^{2}+3 m^{2}-4}{\left(m^{2}-4 n^{2}\right) M}\right)
\end{aligned}
$$

where $M=\left(m^{2}-4+4 n^{2}\right)^{2}-(4 m n)^{2}$.

\section{EXPERIMENTAL CONFIGURATION}

Following the considerations of the previous sections, it is apparent that the primary ingredient necessary for the geometric phase gate is the Hamiltonian (3). As already discussed in section II, the harmonic oscillator is a phase-controllable laser which illuminates both the qubits (Figure 1). The displacement terms in (3) which realize the geometric path of the laser in phase space can be performed using standard quantum optical methods [26]. We thus describe in more detail the atomic configuration that would realize the Hamiltonians.

Consider two qubits, realized for example via cold atoms in traps [27]. A circularly polarized laser pulse is detuned from the atomic resonance transition between one of the hyperfine ground states and an excited state is incident on the atoms $[28,29]$. The Hamiltonian describing the composite system within the single mode and rotating wave approximation $[30-32]$ is given by

$$
H=H_{\text {light }}+H_{\text {atom }}+H_{\text {atom-light }}
$$

where

$$
\begin{aligned}
H_{\text {light }} & =\hbar \omega a^{\dagger} a \\
H_{\text {atom }} & =E_{c}|c\rangle\left\langle c\left|+E_{d}\right| d\right\rangle\left\langle d\left|+E_{\bar{c}}\right| \bar{c}\right\rangle\langle\bar{c}| \\
H_{\text {atom-light }} & =g\left(a|\bar{c}\rangle\left\langle c\left|+a^{\dagger}\right| c\right\rangle\langle\bar{c}|\right)
\end{aligned}
$$

Here, $c$ and $d$ are the logical states of the qubit, and $\bar{c}$ is an excited state. These transition occurs with amplitude $g$, and $E_{c, d, \bar{c}}$ are the energies of the levels involved.

Assuming that the population of the atoms found in the excited state is negligibly small, the dynamics of the excited state can be adiabatically eliminated. The resulting effective Hamiltonian describing dynamics of the ground states are

$$
H_{\mathrm{eff}}=G\left(\sigma^{z}+1\right) a^{\dagger} a
$$

where $\Delta=E_{\bar{c}}-E_{c}-\hbar \omega_{0}, \sigma^{z}=|c\rangle\langle c|-| d\rangle\langle d|$ and

$$
G=\frac{g^{2}}{\Delta}
$$

There is an additional energy offset to the bare photon energy $\hbar \omega$, and thus we have

$$
\hbar \omega_{0}=\hbar \omega+G .
$$

We now compare our results with experimental parameters. The primary constraints are the quality of the cavity and spontaneous emission of the atoms, which adds a decoherence timescale which the operation must be completed within. For example, using experimental parameters associated with ${ }^{85} \mathrm{Rb}$, we have $(g, \kappa, \gamma) / 2 \pi=$ $(16,1.4,3) \mathrm{MHz}$, where $\kappa$ is the cavity field decay rate and $\gamma$ is the spontaneous emission rate [33]. The effective decoherence rate due to spontaneous emission is given by $[34]$

$$
\Gamma_{\mathrm{eff}}=\frac{\gamma g^{2}}{\Delta^{2}}
$$

The effects of spontaneous emission may therefore be suppressed by choosing a detuning $\Delta>g$. Taking $\Delta=2 g$, we then obtain parameters $\left(G, \kappa, \Gamma_{\text {eff }}\right) / 2 \pi=$ $(8,1.4,0.75) \mathrm{MHz}$. As the timescale of the geometric phase gate is determined by $G$ in (13) we see that the operation may be completed within the decoherence times set by the cavity and spontaneous emission. Decoherence/noise can be induced in the experimental implementation of our scheme due to two major factors, imperfect coherent source and time required for the double interaction of light and qubit to execute the gate. So in order to minimize the noise, fast implementation of protocol is essential and demands repetition rate much faster than trap frequency of qubit. For the case of ion trapped systems, obtaining such coherent sources are potentially problematic. But recently it has been shown that the high repetition rate lasers are possible with fibre technology [35], that can help to boost up the speed of gate. Also our scheme does not rely on the perfect resonant coupling ( $\pi$ transitions) so the imperfectness in coherent source can be overlooked for the time being until better options are ready.

\section{CONCLUSION}

We have reexamined the two qubit geometric phase gate for two qubits interacting with a common bosonic mode. The association of geometric phases with each qubit produces an entangled state between qubits. The 
requirement is the presence of a controllable coherent light source which traverses certain geometry in phase space, and an ac Stark shift interaction. The geometric phase derived here uses only the ac Stark shift in the Hamiltonian, without the state-dependent forces that are usually assumed. While in our example solution we used a sinusodial control of the optical field, this can be generalized to other forms. Our formulation may be more useful in cases where an ac Stark shift is more conveniently implemented, rather than state-dependent forces. This formulation could be generalized to other systems, such as quantum dots placed in a microcavity [16]. Another application is to atomic BEC systems, where an ac Stark shift is readily realized via coupling of cavities to optical transitions of atoms [36].

\section{REFERENCES}

[1] A. Barenco, D. Deutsch, A. Ekert, and R. Jozsa, Phys. Rev. Lett 74, 4083 (1983).

[2] C. H. Bennett, G. Brassard, C. Crepeau, R. Joza, A. Peres, and W. K. Wootters, Phys. Rev. Lett 70, 1895 (1993).

[3] C. H. Bennett and S. J. Wiesner, Phys. Rev. Lett 69 , 2881 (1992).

[4] A. K. Ekert, Phys. Rev. Lett 67, 661 (1991).

[5] T. D. Ladd, F. Jelezko, R. Laflamme, Y. Nakamura, C. Monroe, and J. L. O'Brien, Nature 464, 45 (2010).

[6] D. P. DiVinenzo, D. Bacon, J. Kempe, G. Burkard, and K. B. Whaley, Nature 408, 339 (2000).

[7] P. Zanardi and M. Rasetti, Phys. Lett. A 264, 94 (1999).

[8] M. Berry, Proc. R. Soc. London, Ser. A 392, 45 (1984).

[9] L.-M. Duan, J. I. Cirac, and P. Zoller, Science 292, 1695 (2001).

[10] J. J. García-Ripoll, P. Zoller, and J. I. Cirac, Phys. Rev. Lett. 91, 157901 (2003).

[11] S. L. Zhu and Z. D. Wang, Phys. Rev. Lett. 91, 187902 (1999).

[12] J. J. García-Ripoll, P. Zoller, and J. I. Cirac, Phys. Rev. A. 71, 062309 (2005)

[13] D. Leibfried et al., Nature 422, 412 (2003).

[14] D. Hanneke, J. P. Home, J. D. Jost, J. M. Amini, D. Leibfried, and D. J.Wineland, Nature Phy. 6, 1453 (2009).

[15] J. Zhang, Y. Yu, and Z. Zhang, JOSA B 28, 1959 (2011).

[16] S. Puri, N. Y. Kim, and Y. Yamamoto, Phys. Rev. B. 85, 241403(R) (2012).

[17] D. Solenov, S. E. Economou, , and T. L. Reinecke, Phys. Rev. B 87, 035308 (2013).

[18] P. Solinas, P. Zanardi, N. Zanghi, and F. Rossi, Phys. Rev. B 67, 121307 (2003).

[19] P. Treutlein, T. W. Hansch, J. Reichel, A. Negretti, M. A. Cirone, and T. Calarco, Phys. Rev. A. 74, 022312 (2006).
[20] W. Xiang-Bin and M. Keiji, Phys. Rev. Lett. 87, 097901 (2001).

[21] A. Ghosh and A. Kumar, Phys. Lett. A. 349, 27 (2006).

[22] Z. S. Wang, C. Wu, X. Feng, L. C. Kwek, C. H. Lai, C. H. Oh, and V. Vedral, Phys. Rev. A 76, 044303 (2007).

[23] G. Falci, R. Fazio, G. M. Palma, J. Siewert, and V. Vedral, Nature 407, 355 (2000).

[24] W. X. Yang and A. X. Chen, Commun. Theor. Phys. 49, 913 (2008).

[25] L. DiCarlo, J. M. Chow, J. M. Gambetta, L. S. Bishop, B. R. Johnson, D. I. Schuster, J. Majer, A. Blais, L. Frunzio, S. M. Girvin, et al., Nature 460, 08121 (2009).

[26] H. A. Bachor and T. C. Ralph, Wiley-VCH. (2004).

[27] D. Schrader et al, Phys. Rev. Lett. 93, 150501 (2004).

[28] M. R. Andrews, M.-O. Mewes, N. J. van Druten, D. S. Durfee, D. M. Kurn, and W. Ketterle, Science 273, 84 (1996).

[29] J. M. Higbie, L. E. Sadler, S. Inouye, A. P. Chikkatur, S. R. Leslie, K. L. Moore, V. Savalli, and D. M. StamperKurn, Phys. Rev. Lett. 95, 050401 (2005).

[30] J. F. Corney and G. J. Milburn, Phys. Rev. A. 58, 2399 (1998).

[31] J. Ruostekoski and D. F. Walls, Phys. Rev. A. 56, 2996 (1997).

[32] G. J. Milburn, J. Corney, E. M. Wright, and D. F. Walls, Phys. Rev. A. 55, 4318 (1997).

[33] P. Maunz, T. Puppe, I. Schuster, N. Syassen, P. W. H. Pinkse, and G. Rempe, Nature 428, 50 (2004).

[34] A. Pyrkov and T. Byrnes, New Journal of Physics 16, 073038 (2014).

[35] M. J. Petrasiunas, M. I. Hussain, J. Canning, M. Stevenson, and D. Kielpinski, Opt. Express 22, 17716 (2014).

[36] M. I. Hussain, E. O. Ilo-Okeke, and T. Byrnes, Phys. Rev. A 89, 053607 (2014). 